\title{
Valores de vitamina D em pacientes do Hospital do Policial Militar — Goiânia - GO
}

\author{
Vitamin D values in patients at Hospital do Policial Militar — Goiânia - GO \\ Valores de la vitamina D en pacientes del Hospital do Policial Militar - Goiânia - GO
}

\author{
Eduarda Mendes Almeida \\ ORCID: https://orcid.org/0000-0003-0067-6927 \\ Pontifícia Universidade Católica de Goiás, Brasil \\ E-mail: duda_almeidinha@hotmail.com \\ Carini Cristina Cavalcanti Silva \\ ORCID: https://orcid.org/0000-0003-4932-5693 \\ Pontifícia Universidade Católica de Goiás, Brasil \\ E-mail: carinipba-1@hotmail.com \\ Sérgio Henrique Nascente Costa \\ ORCID: https://orcid.org/0000-0002-4225-6368 \\ Pontifícia Universidade Católica de Goiás, Brasil \\ E-mail: sergionascente17@gmail.com \\ Graziela Torres Blanch \\ ORCID: https://orcid.org/0000-0002-9205-0325 \\ Pontifícia Universidade Católica de Goiás, Brasil \\ E-mail: gblanch@pucgoias.edu.br
}

\begin{abstract}
Resumo
Objetivo: Apresentar os índices de alterações de vitamina D em pacientes do município de Goiânia, Goiás. Metodologia: Para a realização desse trabalho, foi feito um levantamento de dados de pacientes do Hospital do Policial Militar do Estado de Goiás, em Goiânia-Go. A análise dos dados foi feita utilizando o microsoft Excel O. Os dados foram então analisados pelo software GraphPadPrism. Após os resultados serem tabulados e as análises serem feitas, foram construídas as tabelas com os valores de vitamina D na população analisada. As análises foram filtradas a partir da amostra inicial de 523 pacientes, divididos por gênero, faixa etária e níveis de vitamina D. Resultados: Observou-se maior número de pacientes com dosagens elevadas de vitamina $\mathrm{D}$, quando comparado com o número de pacientes com dosagens normais ou inferiores ao valor de referência. Conclusão: Foi concluído que as pessoas estão cada vez mais distantes das fontes de vitamina $\mathrm{D}$, e com isso, a saúde da população está sendo prejudicada, principalmente das mulheres.
\end{abstract}

Palavras-chave: Vitamina D; Hipovitaminose; Hipervitaminose.

\begin{abstract}
Objective: To present the indices of vitamin D alterations in patients in the city of Goiânia, Goiás. Methodology: In order to carry out this work, a data survey was carried out from patients at the Hospital do Policial Militar do Estado de Goiás, in Goiânia-Go. Data analysis was performed using Microsoft Excel O. Data were then analyzed using GraphPadPrism software. After the results were tabulated and the analyzes carried out, tables with vitamin D values in the analyzed population were constructed. Analyzes were filtered from the initial sample of 523 patients, divided by gender, age and vitamin D levels. Results: There was a greater number of patients with high doses of vitamin D, when compared to the number of patients with doses normal or below the reference value. Conclusion: It was concluded that people are increasingly distant from sources of vitamin D, and with this, the health of the population is being harmed, especially women.
\end{abstract}

Keywords: Vitamin D; Hypovitaminosis; Hypervitaminosis.

\section{Resumen}

Objetivo: Presentar los índices de alteraciones de la vitamina D en pacientes de la ciudad de Goiânia, Goiás. Metodología: Para la realización de este trabajo se realizó una encuesta de datos a pacientes del Hospital do Policial Militar do Estado de Goiás, en Goiânia-Go. El análisis de datos se realizó usando Microsoft Excel O. Luego, los datos se analizaron usando el software GraphPadPrism. Luego de tabular los resultados y realizar los análisis, se construyeron tablas con los valores de vitamina D en la población analizada. Los análisis se filtraron a partir de la muestra inicial de 523 pacientes, divididos por sexo, edad y niveles de vitamina D. Resultados: Hubo un mayor número de pacientes con dosis altas de vitamina $\mathrm{D}$, en comparación con el número de pacientes con dosis normales o inferiores a las valor de referencia. Conclusión: Se concluyó que las personas están cada vez más alejadas de las fuentes de vitamina D, y con ello se está perjudicando la salud de la población, especialmente de las mujeres.

Palabras clave: Vitamina D; Hipovitaminosis; Hypervitaminosis. 


\section{Introdução}

A vitamina D é um hormônio esteroide produzida in vivo, ela é lipossolúvel, e obtida através da alimentação, sendo que essa via supre em torno de 10 a $20 \%$ das necessidades essenciais do organismo. Além da vitamina D produzida pelo indivíduo, há ainda a formação da vitamina D pela exposição ao sol, que é responsável por 80 a $90 \%$ da vitamina que o corpo necessita, isso acontece através dos raios UVB, ao incidirem sobre a pele, transformam uma pró-vitamina em pré-vitamina D3 (e depois em vitamina D3).

Na pele são produzidos mais de $90 \%$ da vitamina D necessária, onde sua pigmentação, exposição ao sol e ingestão de cálcio influenciam nas concentrações circulantes desta vitamina (Barbosa Kratz, D., Soares Silva, G. \& Tenfen, 2018).

A vitamina D é dividida em dois tipos: Colecalciferol (vitamina D3), proveniente da síntese em animais e Ergocalciferol (vitamina D2) que é de origem vegetal. Ambas fazem parte dos mesmos processos biológicos e das mesmas vias de metabolização, com potências biológicas equivalentes (Pedrosa, \& Castro, 2005). De acordo com Holick (2004) são considerados quadros de deficiência níveis de 25-hidroxicolecalciferol (25(OH) D) abaixo de $20 \mathrm{ng} / \mathrm{mL}$, e de insuficiência, abaixo de $32 \mathrm{ng} / \mathrm{mL}$ (Rafaelli, et al., 2015). As duas formas, podem ser adquiridas através da alimentação e, além disso, a D3 pode ser produzida pelo corpo através do colesterol que é ingerido na dieta, porém este processo necessita de luz do sol. Tanto a D2 quanto a D3 são formas inativas da Vitamina D. A forma ativa é calcitriol (Inda Filho, Jose Melamed, Leora, 2013).

A falta da vitamina D causa no geral uma série de problemas de saúde, pois, ela tem uma grande importância na homeostase do organismo, por meio de sua ação nos ossos, glândulas paratireoides, nos rins e no intestino. Nesses tecidos, ela possui uma forma ativa $(1,25-\mathrm{OH} 2 \mathrm{D})$, que atua ligando-se a um receptor nuclear específico, que pertence à família de receptores dos hormônios esteroides e tireoidianos. Entretanto, esse receptor, bem como a enzima que transforma a 25(OH) D na forma ativa da vitamina D, foram identificados em outros tecidos (Barbosa Kratz, Soares Silva, \& Tenfen, 2018). A forma $25(\mathrm{OH}) \mathrm{D}$ ou a forma ativa 1,25( $\mathrm{OH}) 2 \mathrm{D} 3$ favorecem o funcionamento das células- $\beta$, do sistema renina-angiotensina e da regulação da gordura corporal (Schuch, Garcia, \& Martini, 2009).

O fígado armazena vitamina $\mathrm{D}$ na forma de calcidiol e quando o organismo tem necessidade, uma parte deste depósito é levado até os rins que farão a transformação em calcitriol, que é realmente a forma ativa da Vitamina D (Percegoni, \& Maria 2014).

Figura 1. Mecanismo de síntese da vitamina D.

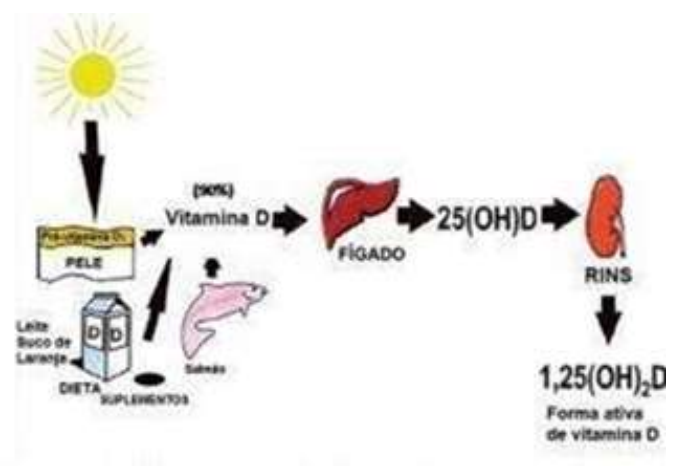

Fonte: Andrade, et al. (2015).

Quando os raios solares penetram na pele, uma parte da luz é transformada em pré-vitamina (7-dehidrocolesterol) e depois na vitamina D3. Então acontece a síntese da vitamina D3 e ela vai para o fígado, onde será convertida em calcidiol, o qual é considerado como indicador dos níveis de vitamina D no organismo. E por último chega-se ao rim, onde a vitamina D. 
A deficiência de vitamina D e suas consequências são consideradas um problema de saúde pública mundial (Cunha, Magalhães, Loureiro, Sant'Ana, Ribeiro, \& Novaes, 2015). No Brasil, estima-se que 77\% da população tenha falta de vitamina, o que é considerado um nível alto, pois, as pessoas estão cada vez mais distantes das fontes de vitamina D, pois, quando elas se protegem do sol com protetor solar, isso faz com que a vitamina não seja absorvida e só a alimentação não é o suficiente, com isso, faz com que ocorra sérios problemas de saúde (Jorge, Cordeiro, Rosa, \& Bianchi, 2018).

A vitamina $\mathrm{D}$ contribui para que aconteça a redução de infecções virais, pelo fato de possuir mecanismos que diminuem o risco de infecção e morte microbiana. Esses mecanismos são divididos em três categorias: barreira física, imunidade natural celular e imunidade adaptativa. Essa vitamina eleva a imunidade celular inata, mas, em partes através da indução de peptídeos antimicrobianos, abrangendo a catelicidina humana, LL-37, pela 1,25-dihidroxivitamina D e defensinas. A vitamina D também aumenta a imunidade celular, entretanto, em parte diminuindo a tempestade de citocinas induzida pelo sistema imunológico inato, o qual origina citocinas pró-inflamatórias e anti-inflamatórias em resposta a infecções virais e bacterianas, como foi observado em pacientes com COVID-19. A vitamina D é um modulador da imunidade adaptativa; 1,25( $\mathrm{OH}) 2 \mathrm{D} 3$ anula as respostas mediadas pela célula T-helper tipo 1 (Th1), limitando a princípio a produção de citocinas inflamatórias IL-2 e interferon gama (INF y). Além do mais, 1,25(OH)2D3 proporciona a produção de citocinas pelas células T-helper tipo 2 (Th2), o que colabora para a melhoria da supressão indireta das células Th1, complementando-a com ações mediadas por vários tipos de células e também a 1,25(OH)2D3 possibilita a indução das células reguladoras T, inibindo assim processos inflamatórios. As concentrações séricas da vitamina $\mathrm{D}$ diminuem no decorrer que a pessoa vai envelhecendo, e isso faz com que aquelas pessoas que estão com uma idade considerada mais avançada, ficam mais vulneráveis e com isso, podem adquirir o COVID-19 ou outra qualquer infecção com mais facilidade, pois, as taxas de mortalidade, mostram que essas pessoas que possuem a idade mais avançada estão se infectando com esse vírus com mais facilidade (Grant, Lahore, Donne. Baggerly, French, Aliano, et al. 2020).

A vitamina $\mathrm{D}$, também é usada para prevenção de várias doenças como: câncer, diminuir a chance de artrite reumatoide, doenças cardiovasculares, interromper processos inflamatórios de doenças autoimunes e até mesmo, resfriados e emagrecimento. No ano de 2011, a Academia Nacional de Medicina dos Estados Unidos, aumentou a recomendação de vitamina D, estipulando o valor de referência de $400 \mathrm{UI} /$ dia até um ano de idade e de $600 \mathrm{UI} /$ dia para crianças e jovens de 1 a 18 anos de idade. A concentração existente de vitamina D no leite materno é de aproximadamente 22 UI/litro e nas fórmulas infantis de cerca $10 \mathrm{mg} / \mathrm{L}$ (400 UI/litro). O Departamento Científico de Nutrologia da SBP sugere a suplementação profilática de $400 \mathrm{UI} /$ dia a partir da primeira semana de vida até os 12 meses, e de $600 \mathrm{UI} / \mathrm{dia}$ dos 12 aos 24 meses, mesmo para as crianças em aleitamento materno restrito, independente da região do país. Para os recém-nascidos, a suplementação oral de vitamina D (400 UI/dia) deve ser estabelecida quando o peso for superior a $1500 \mathrm{~g}$ e possuir tolerância à nutrição enteral. Os grupos considerados de riscos são idosos, lactantes, gestantes, pacientes com hiperparatiroidismo, raquitismo, doenças inflamatórias entre outros, a avaliação do estado nutricional relativo à vitamina $\mathrm{D}$, é recomendada a dose mínima diária de 600 UI, controlando as concentrações séricas de $25(\mathrm{OH}) \mathrm{D}$, e sempre que necessário, é preciso ir reajustando a dose (Pediatria, de. 2014).

A vitamina D é de extrema importância para o tecido ósseo e também para prevenção de doenças crônicas, como por exemplo: diabetes tipo 1, esclerose múltipla e outras. O calcitriol (forma ativa da vitamina D) é responsável pelo comando dos níveis plasmáticos de cálcio no sangue, além disso, também exerce a função imunomoduladora e induz a secreção de insulina. A calcinose é a manifestação mais comum nas situações de intoxicação por vitamina D. Ela constitui-se na ampla mineralização de tecidos moles; com hipervitaminose D a mineralização das artérias originando um intenso empenho do sistema cardiovascular (Caserta, Antunes, \& Rocha, 2016). Quando ocorrem casos de intoxicação pela vitamina D, os metabólitos (principalmente a 1,25- vitamina D) aparecem em níveis elevados, é atingida a capacidade das proteínas 
transportadoras da vitamina D e agravada a capacidade de metabolização e excreção hepática. A intoxicação aguda pela vitamina D decorre com hipercalcemia e hiperfosfatemia, com isso, a hipercalcemia pode levar o paciente a desenvolver nefrocalcinose, hipercalciúria e hipertensão de causa renal. Com essa intoxicação da hipercalcemia, o sistema nervoso central sofre alterações como, convulsões, tetania, hiperexcitabilidade neuromuscular, parestesias e coma (Santa, Sf., et al., 2008). A vitamina D é muito conhecida por exercer a função no desenvolvimento e na manutenção do tecido ósseo e, contudo, pela manutenção da homeostase normal do cálcio e do fósforo. Mas, evidências atuais aconselha o envolvimento dessa vitamina em vários processos celulares vitais, como: diferenciação e proliferação celular, secreção hormonal (como por exemplo, o da insulina), igualmente no sistema imune e em outras várias doenças crônicas não transmissíveis (Caserta L, Antunes J, Rocha $\mathrm{Da}, 2016)$. $\mathrm{O}$ tratamento da intoxicação pela vitamina $\mathrm{D}$ se dá pela a retirada rápida da fonte exógena e também a correção das alterações do metabolismo fosfocálcico (Santa, et al., 2008).

A principal causa de falta de vitamina $\mathrm{D}$, é a baixa exposição ao sol e isso pode levar o indivíduo a desenvolver uma hipovitaminose. A hipovitaminose D em crianças leva ao retardo do crescimento e ao raquitismo, em adultos leva à osteomalácia, ao hiperparatiroidismo secundário e consequentemente ao aumento da reabsorção óssea, favorecendo assim a perda de massa óssea e ao desenvolvimento de osteopenia e osteoporose. Fraqueza muscular também pode ocorrer, o que contribui para elevar ainda mais o risco de quedas e de fraturas ósseas em pacientes com baixa massa óssea. O tratamento disponível para a hipovitaminose D é o colecalciferol ou vitamina D3, pois, este metabólito foi o que mostrou maior eficiência. O ergocalciferol ou vitamina D2 também é uma opção de suplemento, porém as pesquisas mostram que, por sua meia-vida ser um pouco inferior à $\mathrm{D} 3$, a posologia deva ser de preferência diária. Além do mais, alguns procedimentos laboratoriais que dosam $25(\mathrm{OH})$ D aprova apenas a $25(\mathrm{OH}) \mathrm{z}$ D3, o que pode trazer consequências ruins no controle dos níveis plasmáticos quando se faz a reposição com vitamina D2. Dessa maneira, mesmo que a suplementação e o tratamento possam ser realizados com os dois metabólitos da vitamina $\mathrm{D}$, deve-se dar preferência para a vitamina D3 por causa de suas vantagens sobre a manutenção de concentrações melhores. As doses para tratamento diversificam de acordo com o grau de deficiência e com a meta a ser atingida (Maeda, et al., 2014).

\section{Metodologia}

Para a realização desse trabalho, foi feito um levantamento de dados de pacientes do Hospital do Policial Militar do Estado de Goiás, em Goiânia-Go. Na tabela original constava o total de 523 pacientes, os quais foram separados por sexo e por idade: de 0 a 19 anos; 20 a 59 anos e $\geq 60$ anos. Os dados foram coletados no período de 3 de janeiro de 2018 a 19 de dezembro de 2018 e selecionados os valores dos níveis sanguíneos de vitamina D. A análise dos dados foi feita utilizando o microsoft Excel ®. Os dados foram então analisados pelo software GraphPadPrism.

Após os resultados serem tabulados e as análises serem feitas, foram construídas as tabelas com os valores de vitamina D na população analisada. As análises foram filtradas a partir da amostra inicial de 523 pacientes, divididos por gênero, faixa etária e níveis de vitamina D.

\section{Resultados e Discussão}

Os valores de referência da vitamina D por faixa etária, estão demonstradas na Tabela 1 e mostra os parâmetros segundo a Sociedade Brasileiras de Patologia, Clínica/Medica Laboratorial e da Sociedade Brasileira de Endocrinologia e Metodologia (Santa Sf, et al., 2008). Foram utilizados esses parâmetros que o estudo foi guiado para a análise dos dados. 
Tabela 1. Valores de referência vitamina D (25-OH), segundo a Sociedade Brasileira de Patologia Clínica/Medicina Laboratorial e da Sociedade Brasileira de Endocrinologia e Metabologia - Método: Quimioluminescência.

\begin{tabular}{ccc}
\hline $\begin{array}{c}\text { Até } 60 \text { anos de } \\
\text { idade }\end{array}$ & Grupo de risco & $\begin{array}{c}\text { Risco de } \\
\text { toxicidade e } \\
\text { Hipercalcemia }\end{array}$ \\
\hline $\begin{array}{c}\text { Superior a } 20,0 \\
\mathrm{ng} / \mathrm{mL}\end{array}$ & $\begin{array}{c}\text { De } 30,0 \text { a } 60,0 \\
\mathrm{ng} / \mathrm{mL}\end{array}$ & $\begin{array}{c}\text { Superior a } \\
100 \mathrm{ng} / \mathrm{mL}\end{array}$ \\
\hline
\end{tabular}

Fonte: De Moraes, et al. (2018).

Quando os dados foram separados por gênero (homens e mulheres, Tabela 2 e 3), foram encontrados um maior número de exames de pacientes do sexo feminino (totalizando 413 pacientes do sexo feminino e 110 pacientes do sexo masculino).

A Tabela 2, demonstra um total de 413 pacientes do sexo feminino. Sendo que $13 \%$ apresentaram valores de inferiores a $20 \mathrm{ng} / \mathrm{mL}$ (alto risco para uma hipovitaminose D), 49\% apresentaram resultados que são considerados normais e $38 \%$ apresentaram valores superiores a $30 \mathrm{ng} / \mathrm{mL}$ (alto risco para uma hipervitaminose D).

Tabela 2. Número e média de pacientes do sexo feminino com resultados alterados no exame de vitamina D.

\begin{tabular}{ccccccc}
\hline $\begin{array}{c}\text { Faixa } \\
\text { etária } \\
\text { Feminino }\end{array}$ & $\begin{array}{c}\text { Inferior } \\
\mathbf{2 0} \\
\text { ng/mL }\end{array}$ & $\begin{array}{c}\text { Média } \\
\mathbf{\pm} \\
\text { DPM }\end{array}$ & $\begin{array}{c}\mathbf{2 0 - 3 0} \\
\mathbf{n g} / \mathbf{m L}\end{array}$ & $\begin{array}{c}\text { Média } \\
\mathbf{\pm} \\
\mathbf{D P M}\end{array}$ & $\begin{array}{c}\text { Superior } \\
\mathbf{3 0} \\
\mathbf{n g} / \mathbf{m L}\end{array}$ & $\begin{array}{c}\text { Média } \\
\mathbf{\pm} \\
\mathbf{D P M}\end{array}$ \\
\hline $0-19$ & 0 & 0 & 10 & 26,5 & 7 & 36,5 \\
& & 0 & & $\pm 2,5$ & & $\pm 5,0$ \\
$20-59$ & 42 & 16,9 & 146 & 25,7 & 107 & 39,5 \\
& & $\pm 2,5$ & & $\pm 3,0$ & & $\pm 2,5$ \\
$\geq 60$ & 12 & 16,8 & 46 & 25,5 & 43 & 40,9 \\
& & $\pm 2,7$ & & $\pm 2,6$ & & $\pm 12,7$ \\
Total & 54 & 33,7 & 202 & 77,7 & 157 & 116,9 \\
\hline
\end{tabular}

Fonte: Dados do Hospital do Policial Militar, Goiânia-GO (2018).

Quando a Tabela 3 foi analisada, foram constados resultados de 110 pacientes do sexo masculino. Sendo que 5,4\% apresentaram valores inferiores a $20 \mathrm{ng} / \mathrm{mL}$ (alto risco de uma hipovitaminose D), 43,6\% apresentaram valores normais e 51\% apresentaram valores superiores a $30 \mathrm{ng} / \mathrm{mL}$ (alto risco de uma hipervitaminose D). 
Tabela 3. Número e média de pacientes do sexo masculino com resultados alterados no exame de vitamina D.

\begin{tabular}{|c|c|c|c|c|c|c|}
\hline $\begin{array}{c}\text { Faixa } \\
\text { etária } \\
\text { masculino }\end{array}$ & $\begin{array}{c}\text { Inferior } \\
20 \\
\text { ng/mL }\end{array}$ & $\begin{array}{c}\text { Média } \\
\pm \\
\text { DPM }\end{array}$ & $\begin{array}{l}20-30 \\
\mathrm{ng} / \mathrm{m} \\
\mathrm{L}\end{array}$ & $\begin{array}{c}\text { Média } \\
\pm \\
\text { DPM }\end{array}$ & $\begin{array}{c}\text { Superior } \\
\mathbf{3 0} \\
\mathrm{ng} / \mathrm{mL}\end{array}$ & $\begin{array}{c}\text { Média } \\
\pm \\
\text { DPM } \\
\end{array}$ \\
\hline 0-19 & 1 & $\begin{array}{c}18 \\
0\end{array}$ & 9 & $\begin{array}{c}24,8 \\
\pm 2,9\end{array}$ & 4 & $\begin{array}{l}36,25 \\
\pm 6,5\end{array}$ \\
\hline $20-59$ & 4 & $\begin{array}{r}17,7 \\
\pm 1,2\end{array}$ & 29 & $\begin{array}{r}25,8 \\
\pm 3,2\end{array}$ & 39 & $\begin{array}{c}43,9 \\
\pm \\
18,6\end{array}$ \\
\hline$\geq 60$ & 1 & $\begin{array}{c}16 \\
0\end{array}$ & 9 & $\begin{array}{c}25,1 \\
\pm 2,7\end{array}$ & 13 & $\begin{array}{c}43,2 \\
\pm \\
19,1\end{array}$ \\
\hline Total & 6 & 51,7 & 48 & 75,7 & 56 & $\begin{array}{c}123,3 \\
5\end{array}$ \\
\hline
\end{tabular}

Fonte: Dados do Hospital do Policial Militar, Goiânia-GO (2018).

Para o estudo dos resultados, partimos da análise da Tabela 1, a qual mostra os valores de referência de vitamina D que são adequados para cada faixa etária. Os valores são divididos em três itens: o primeiro item refere-se aos pacientes até 60 anos, estes tem que apresentar resultados superiores a 20,0 ng/mL, o segundo item está se referindo aqueles pacientes que são de grupo de risco, devem apresentar resultados de 30,0 a 60,0 ng/mL e o terceiro item, já se refere aqueles pacientes que se enquadram no grupo de risco de toxicidade e hipercalcemia, pois apresentando os resultados dos exames superiores a 100 ng/mL (De Moraes Acf, et al., 2018).

As Tabelas 2 e 3 foram divididas em gêneros (feminino e masculino) e faixas etárias, com os resultados dos exames de vitamina D que estão nos arquivos do Hospital do Policial Militar do município de Goiânia do estado de Goiás.

Maeda et al, (2014) estudaram populações de outros municípios e estados, foi divulgado no ano de 2014 e ele relata que existem altos indices de pessoas com hipovitaminose. Esse artigo consta um total de 4.375 pessoas que tiveram seus exames de vitamina D estudados, foram separados em grupos de risco e aqueles que não se enquadram no grupo de risco, também foram separados em homens e mulheres. Essas pessoas são de São Paulo — SP, Vitória- ES, Belo Horizonte-MG, Indaiatuba - SP, Rio de Janeiro — RJ e Recife — PE. Já no estudo que foi realizado, a população do município de Goiânia — GO, uma quantidade pequena de pessoas estão com pouca suplementação de vitamina D e grande quantidade estão com uma alta suplementação dessa vitamina, poucas pessoas estão com o nível dessa vitamina normal, o que pode ser algo preocupante e pode ser considerado um estado de alerta. É importante frisar que nas duas pesquisas, as que mais se sobressaíram foram os pacientes do sexo feminino.

Segundo Unger (2009), no estado de São Paulo no ano de 2009, foram estudadas 603 pessoas (118 homens e 485 mulheres). O sexo feminino também foi predominante com uma porcentagem de 80,4 \% de participação enquanto os homens foram apenas 19,6\%. Essas pessoas que se dispuseram a ser voluntárias, foram divididas em 4 grupos, para que fosse possível fazer uma comparação de níveis séricos de 25 vitamina $\mathrm{D}$ entre inverno e verão. O grupo 1 foi de indivíduos com níveis séricos de 25 vitamina $\mathrm{D}<30 \mathrm{ng} / \mathrm{mL}$, independente da estação do ano (n=72); o grupo 2 foi dos indivíduos com níveis séricos de 25 vitamina $\mathrm{D}<30 \mathrm{ng} / \mathrm{mL}$ após o inverno e $\geq 30 \mathrm{ng} / \mathrm{mL}$ após o verão ( $\mathrm{n}=88$ ); o grupo 3 fó daqueles indivíduos com níveis séricos de 25 vitamina D sempre $\geq 30 \mathrm{ng} / \mathrm{mL}$, independente da estação do ano (n=43) e por fim, o grupo 4 foi dos indivíduos com níveis séricos de 25 vitamina $\mathrm{D} \geq 30 \mathrm{ng} / \mathrm{mL}$ após o inverno e $<30 \mathrm{ng} / \mathrm{mL}$ após o verão (n=4). Quando foi comparado o resultado do presente estudo com essa pesquisa citada acima, achou-se que novamente o sexo feminino se sobressaiu e as duas 
pesquisas mostraram que existem um alto índice de indivíduos com hipovitaminose e poucos com os níveis séricos de vitamina D, considerados normais.

Quando a hipervitaminose é citada, há autores como Corrêa et al., (2016), afirma que as doenças endócrinas como hiperparatiroidismo primário, síndrome NEM I e II, doenças malignas como por exemplo o linfoma de Hodgkin e linfoma não Hodgkin, doenças granulomatosas como por exemplo sarcoidose e tuberculose podem ser associadas com hipervitaminose. Apesar que a maioria dos casos de hipercalcemia, podem ser causadas por hiperparatiroidismo primário ou malignidade, um diagnóstico diferente que pode ser excluído é o da hipervitaminose D.

A intoxicação por hipervitaminose D é rara de acontecer, mas, é considerada ser grave para a saúde do indivíduo. O tratamento com vitamina D deve ter acompanhamento. Contudo, quando se tem um quadro clínico suspeito e um estado de hipercalcemia, com o nível do hormônio da paratireoide normal ou baixo, o diagnóstico de intoxicação pela vitamina $\mathrm{D}$, tem que ser uma das sugestões de suspeita e quando esse nível se normaliza, os sintomas são aliviados e isso pode prevenir uma lesão renal aguda (Longo et al., 2016). Nesse estudo, foram encontrados 157 pacientes do sexo feminino e 56 do sexo masculino, apresentando quadro de hipervitaminose, o que parece ser derivada de um excesso de suplementação, já que algumas pessoas fazem essa suplementação sem orientação médica.

\section{Conclusão}

Frente aos dados apresentados, nota-se que as pessoas estão cada vez mais distantes das fontes de vitamina $\mathrm{D}$, e com isso, a saúde da população pode estar está sendo prejudicada, principalmente das mulheres. Muitos indivíduos quando são expostos ao sol, estão protegidos com protetores solar ou roupas que não deixam os raios UVB penetrarem na pele e com isso, acabam deixando faltar essa vitamina ou então tomam uma suplementação por conta própria, sem acompanhamento médico. Por fim, acabam arriscando sua saúde por conta da falta ou excesso dessa vitamina. Assim, trona-se necessário políticas de educação em relação aos benefícios do acompanhamento dos valores da vitamina D para a saúde dos indivíduos, fomentando profissionais da saúde a monitorarem e orientarem a população. Como próximos passos o acompanhamento dos valores desata vitamina deve ser feito também em menores de 18 anos, para que possamos ter um perfil mais amplo e para que eventuais deficiências ou excessos possam ser sanados.

\section{Referências}

Andrade, P. C. O., Castro, L. S., Lembetucci, S. M. \& Larcerda, B. M. I. P. et al. (2015). Diet, sun exposure, and dietary supplementation: effect on serum levels of vitamin D. Rev Médica Minas Gerais. 25(3):432-7.

Barbosa Kratz, D., Soares Silva, G. \& Tenfen, A. (2018). Deficiência de vitamina D (250H) e seu impacto na qualidade de vida: uma revisão de literatura. Rev Braseileira Análises Clínicas. 50(2):118-23.

Caserta, L., Antunes, J. \& Rocha, D.A. (2016). Consumo Excessivo De Produtos Vitamínicos: Uma revisão. Rev UNINGA. 47:84-8.

Cunha, K. A., Magalhães, E. I. D. S., Loureiro, L. M. R., Sant'Ana, L. F. D. R., Ribeiro, A. Q. \& Novaes, J. F. (2015). Calcium intake, serum vitamin D and obesity in children: Is there an association? Rev Paul Pediatr. 33(2):222-9.

De Moraes, A. C. F., Maeda, S. S., Batista, M. C., Lazaretti Castro, M., Vasconcellos, L. de S. \& Madeira, M., et al. (2018). Posicionamento Oficial da Sociedade Brasileira de Patologia Clínica/ Medicina Laboratorial e da Sociedade Brasileira de Endocrinologia e Metabologia. J Bras Patol Med Lab. 53(6):377—81.

Grant, W. B., Lahore, H., M. C. Donne. I. I. S. L., Baggerly, C. A., French, C. B., Aliano, J. L., et al. (2020). Evidence that Vitamin D Supplementation Could Reduce Risk of Influenza and COVID-1 Infections and Deaths. Nutrients. 12(4):988.

Holick, M. F. (2004). Vitamin D: importance in the prevention of cancers,type 1 diabetes,heart disease and osteoporosis. Am J Clin Nutr.79(5):362 - 71.

Inda Filho, A. J., Melamed, M. L. (2013). Vitamin D and kidney disease: what we know and what we do not know. J Bras Nefrol. Orgão Of Soc Bras e LatinoAmericana Nefrol. 35(4):323-31.

Jorge, A. J. L., Cordeiro, J. R., Rosa, M. L. G. \& Bianchi, D. B. C. (2018). Deficiência da Vitamina D e Doenças Cardiovasculares. Int J Cardiovasc Sci. 31(4):422-32.

Longo, B., Corrêa, B. B., Lagana, C., Robl, M., Besen, D. C., Manosso, K. Z. B., et al. (2016) Intoxicação Por Calcitriol Após Tratamento De Hipoparatireoidismo 
Research, Society and Development, v. 10, n. 13, e430101321384, 2021

(CC BY 4.0) | ISSN 2525-3409 | DOI: http://dx.doi.org/10.33448/rsd-v10i13.21384

Transitório. Rev Médica da UFPR.3(2):95-7.

Maeda, S. S., Borba, V. Z. C., Camargo, M. B. R., Silva, D. M. W., Borges, J. L. C., Bandeira, F., et al. (2014). Recomendações da Sociedade Brasileira de Endocrinologia e Metabologia (SBEM) para o diagnóstico e tratamento da hipovitaminose D. Arq Bras Endocrinol Metabol. 58(5):411—33.

Pediatria, S. B. de. (2014). Deficiência de vitamina D em crianças a adolescentes, In: Sociedade Brasileira de Pediatria, $1132-41$.

Pedrosa, M. A. C. \& Castro, M. L. (2005). Papel da vitamina D na função neuro-muscular. Arq Bras Endocrinol Metabol. 49(4):495-502.

Percegoni, N. \& Maria, de A. C. J. (2014). Obesidade Metabolismo Alimentação VD. Vitamina D, sobrepeso e obesidade-Uma revisão. HU Rev [Internet].40(3 e 4):209-19

Rafaelli R. A., Nomura, P. R., Figueira, F., Santos, I., Silva, L. \& Venturini, D. (2015). Influência da vitamina D nas doenças endocrinometabólicas. Semin Ciências Biológicas e da Saúde.36(1):333-48.

Santa, S. F., Chicamba, V., Valente R., Ventura L., Ramos, J., Barata, D. \& Fonseca, G. R. S. (2008). Coma Hipocalcémico por Hipervitaminose D. Acta Pediátrica Port.39(2):75-8.

Schuch, N. J., Garcia, V. C. \& Martini, L. A. (2009). Vitamina D e doenças endocrinometabólicas. Arq Bras Endocrinol Metabol.53(5):625—33.

Unger, M. D. (2009). Determinação dos níveis séricos de vitamina D em uma amostra de indivíduossaudáveis da população brasileira. 